\title{
1 Achtsamkeit und achtsamkeitsbasierte Interventionen
}

\author{
Niko Kohls
}

\section{Psychologische Definition von Achtsamkeit}

Obwohl das Konzept der Achtsamkeit in den meisten spirituellen, kontemplativen und meditativen Systemen - zumindest implizit - eine wichtige Rolle spielt, wird der Begriff häufig mit den buddhistischen Weisheitslehren verbunden. Denn in diesem Kontext beschreibt Achtsamkeit im expliziten Einklang mit dem epistemologischen Kern der buddhistischen Weltanschauung eine spezifische Form der Bewusstseinskultivierung, obwohl sich konzeptionelle und epistemologische Verbindungen zur Achtsamkeit beispielsweise auch in den theistischen Religionen und so beispielsweise auch in der christlichen Mystik finden lassen (Kohls 2004). Während Bewusstseinskultivierung in den spirituellen Traditionen durch Einüben von meditativen und kontemplativen Praktiken und Techniken erreicht wird, hat sich im Zusammenhang mit säkularisierten Ansätzen zunehmend der im Kontext der integrativen Medizin häufig verwendete angloamerikanische Terminus „Mind-Body-Practices“ - darunter fällt z.B. Qi-Cong, Thai-Chi oder körperorientierte Psychotherapie - eingebürgert (Kohls 2004; Walach et al. 2012).

Den Zustand der Achtsamkeit kann man dabei als eine wichtige psychologische Funktion all dieser Techniken - seien sie spirituell oder säkular - ansehen (Sauer et al. 2011). Eine Fokussierung des Bewusstseins auf das gegenwärtige Erleben - sowohl in Bezug auf das psychologische Milieu intérieur als auch milieu extérieur - mit einer annehmenden, positiven Grundhaltung, ohne dabei die Wahrnehmungsinhalte kognitiv zu bewerten (Sauer et al. 2011). In der Terminologie der akademischen Psychologie wird „Achtsamkeit“ daher als ein spezifischer, trainierbarer Bewusstseinszustand verstanden, der auf das direkte und nicht-wertende Gewahrsein dessen abzielt, 
was in jedem Augenblick geschieht (Bishop 2004). Zum gegenwärtigen Zeitpunkt ist jedoch die Frage ungeklärt, ob Achtsamkeit als ein eindimensionales Konstrukt zu verstehen ist, oder ob es aus zwei oder mehreren Unterfacetten - wie beispielsweise einer „kognitiven Präsenz-“ und einer „emotionalen Akzeptanzkomponente“ besteht (Kohls et al. 2009; Sauer et al. 2013).

\section{Wirkmechanismen von Achtsamkeit}

Dass Achtsamkeit positive Auswirkungen auf Stresswahrnehmung, Wohlbefinden, Lebensqualität und Gesundheitsverhalten haben kann, belegen viele Studien eindeutig (Grossman et al. 2004; Eberth u. Sedlmeier 2012).

Gegenwärtig besteht jedoch kein vollständiger Konsens über die Wirkfaktoren von Meditation im Allgemeinen und achtsamkeitsbasierter Meditation im Speziellen. Diskutiert werden jedoch Modelle, die sich von ihrem Erklärungsgehalt teilweise überschneiden. So wird angenommen, dass durch das Üben von Meditation im Sinne einer introspektiven Schulung eine verbesserte Emotionsregulation (Arch u. Craske 2006), eine bessere Allokation von Aufmerksamkeitsressourcen (Lutz et al. 2008) realisiert werden kann.

Ein von Shauna Shapiro und Kollegen entwickeltes theoretisches Modell - die sogenannte IAA-Theorie - postuliert drei zusammenhängende Bestandteile von Achtsamkeit (Shapiro 2006):

1. Intention (englisch Intention),

2. Aufmerksamkeit (englisch Attention) und

3. Einstellung (englisch: Attitude).

Der Theorie zufolge bewirkt ein intentionales Fokussieren (Intention) der Aufmerksamkeit (Attention) mit einer nichtbewertenden Haltung der Offenheit (Attitude) eine spezifische Veränderung der Wahrnehmung, die sie „Reperceiving“ nennen. Reperceiving wird dabei als die entwickelbare Fähigkeit im Sinne eines übergeordneten Prozesses verstanden, durch die es möglich wird, Erfahrungen unter einem neuen Blickwinkel zu sehen.

\section{Neurobiologische Grundlagen von Achtsamkeit}

Von dem zunehmenden Interesse innerhalb der Neurobiologie und Hirnforschung, die neuronalen Korrelate von veränderten Bewusstseinszuständen empirisch zu untersuchen, hat vor allem die Meditationsforschung profitiert (Vaitl et al. 2005; Cahn u. Polich 2006). War die frühe Forschung noch stark auf die transzendentale Meditation fokussiert, hat die Erforschung von Zuständen der Achtsamkeit in den letzten Jahren - wohl nicht zuletzt aufgrund der zunehmenden Popularität von achtsamkeitsbasierten Verfahren - zunehmend an Bedeutung gewonnen.

Eine der bekanntesten frühen Studien wurde von Richard Davidson und Kollegen an der Universität Wisconsin durchgeführt (Davidson et al. 2003). Dabei wurde das EEG-Muster sowie die Influenza-Antikörper-Konzentration im Blut von 25 Teilnehmern eines MBSR-Programms mit 16 Personen einer Warteliste-Kontrollgruppe vor und nach der Intervention verglichen. Es zeigte, dass in der MBSR-Gruppe nicht nur die Aktivierung im linken Frontlappen - eine Gehirnregion deren Aktivierung mit positiven Affekt assoziiert wird - im Vergleich zur Kont- 
rollgruppe erhöht war, sondern auch der Immunstatus - bestimmt durch Antikörperkonzentration für eine Influenzaimpfung - besser war. In dieser Studie konnte erstmals ein direkter Zusammenhang zwischen dem linksfrontalen Aktivierungsmuster und der Antikörperkonzentration nachgewiesen werden.

Etliche Studien haben jedoch aufzeigen können, dass achtsamkeitsbasierte Verfahren nicht nur Gehirnprozesse unter funktionalen Gesichtspunkten verändern können, sondern, dass darüber hinaus auch die Hirnmorphologie im Sinne der Neuroplastizität strukturell verändert werden kann (Lazar et al. 2005; Hölzel et al. 2008). Beispielsweise fanden Hölzel und Kollegen dass die Konzentration an den üblicherweise eher außen liegenden grauen Zellen bei Personen mit regelmäßiger VipassanaMeditationserfahrung in den Hirnregionen, die üblicherweise während meditativen Übungen involviert sind, stärker ausgeprägt war als bei vergleichbaren Personen ohne regelmäßige meditative Praxis.

In der Summe sprechen die Studienergebnisse dafür, dass sich das Gehirn von meditierenden Menschen schon nach einem relativ kurzen Zeitraum sowohl funktional als auch strukturell im Sinne der Neuroplastizität zu verändern beginnt. Obwohl Veränderungen nicht nur beim Meditieren, sondern bei fast allen Lernprozessen beispielsweise zu Lernen wie man jongliert - auftreten, spricht doch einiges dafür, dass die Effekte von Meditation im Sinne der systematischen Schulung der eigenen Gegenwartswahrnehmung spezifische Wirksamkeit entfalten können.

\section{Achtsamkeit und die Psychologie der Zeitwahrnehmung}

Die Fähigkeit, den gegenwärtigen Moment möglichst direkt und unverfälscht in einer akzeptierenden Weise zu erleben, erscheint bei oberflächlicher Betrachtung als einfache, wenn nicht sogar gehaltlose Angelegenheit. Denn es scheint, dass wir Menschen - weil wir eben in einen scheinbar kontinuierlich dahinfließenden Zeitstrom eingebettet sind - den gegenwärtigen Moment - den wir mit dem Begriff ,Jetzt“ bezeichnen - bei vollem Bewusstsein stets als einen flotierenden phänomenalen Ereignispunkt wahrnehmen, der von Vergangenem und Zukünftigem umrahmt wird (Wittmann 2009; 2011; 2013).

Jedoch ist die Einübung des Bewusstseinszustands der Achtsamkeit - nicht nur in der meditativen Versenkung, sondern vor allem in der Verankerung im Alltag - alles andere als trivial. Um dies zu verstehen, muss man sich ein wenig mit der neurobiologischen Architektur des Gehirns beschäftigen, die es uns ermöglicht, über das autobiographische Gedächtnis sowohl die Vergangenheit in kondensierter Form unseres Erlebten zu vergegenwärtigen, als auch unser gegenwärtiges Handel auf ein bestimmtes in der Zukunft liegendes, angestrebtes Ziel hin auszurichten. Diese kognitiv-emotionalen Rückgriffe in das autobiographische Gedächtnis sowie das antizipierende, auf einen zukünftigen Zustand bezogene Handeln stellen aus evolutionsbiologischer Sicht äußerst wirkungsvolle Mechanismen zur Verfügung, mit deren Hilfe über Lernprozesse komplexe Problemlöse- und Planungsfähigkeiten realisiert werden können. Andererseits führen diese Strategien aufgrund der gebotenen Optimierung der Wahrnehmungsprozesse naturgemäß auch zu einer reduzierten Genauigkeit des Gegenwartserlebens zugunsten von effizienten Mustererkennungsprozessen: Vereinfacht gesagt, es werden für die Gegenwartswahrnehmung immer nur so viele sensorischer Reize verarbeitet, wie für schnelle und hinreichend akkurate Ent- 
scheidungsprozesse notwendig sind. Auf der Crundlage von früheren Lernerfahrungen herausgebildete mentale Repräsentationskategorien können diese Prozesse auf Kosten der Wahrnehmungsgenauigkeit beschleunigen. Achtsamkeit kann auf diesem Hintergrund auch als Versuch beschrieben werden, die individuellen erlernten (und partiell auch angeborenen) Stereotypen und Vorurteile bewusst unter Kontrolle zu halten. Dies führt zu einer weniger individualistischen Sichtweise, wodurch verhindert wird, dass die Wahrnehmung zu stark „subjektiv eingefärbt“ ist. Interessant ist in diesem Zusammenhang die Studie, die die neuronalen Korrelate von Achtsamkeit mit Hilfe der funktionellen Magnetresonanztomographie (fMRT) untersucht hat (Farb et al. 2007). Die Ergebnisse lagen nahe, dass durch achtsamkeitsbasierte Interventionen das Selbstmodell von Meditierenden so verändert werden kann, dass sie die Hirnareale, die für die gegenwärtige Selbst- und Körperwahrnehmung zuständig sind, von den Arealen, die für ihr autobiographisches Gedächtnis und damit für ihr biographisches Selbstbild zuständig sind, abgekoppelt werden können.

\section{Achtsamkeit als Reaktion auf Belastung und risikobehaftetes Gesundheitsverhalten}

Die zunehmende Popularität von Achtsamkeit kann möglicherweise auch im Zusammenhang mit der Zunahme an psychischer Belastung und einem wenig ausgeprägten Gesundheitsverhalten im Sinne einer Gegenreaktion gesehen werden. So zeigen 2009 erhobene repräsentative Umfragedaten von Techniker-Krankenkasse, FAZ-Institut und Forsa beispielsweise auf, dass vier von fünf Deutschen - also 8o\% der Bevölkerung - ihre Lebensumstände als „stressig“ erleben. Jeder dritte Befragte gab bei der Umfrage sogar an, „unter Dauerdruck zu stehen“ (FAZ-Institut \& Techniker-Krankenkasse, 2009). Hauptursachen waren dabei Jobstress, finanzielle Sorgen und Ärger im Straßenverkehr. Das dieses vermeintlich nur subjektiv relevante Stresserleben eine große volkswirtschaftliche Dimension besitzt, wird deutlich, wenn man sich vergegenwärtigt, dass in der deutschen Wirtschaft 2008 fast zehn Millionen Fehltage wegen „Burnout-Symptomen“ zustande kamen. Als besonders gefährdet erweisen sich dabei Menschen in sozialen Berufen, Beschäftigte in Sandwich-Positionen, Schichtarbeiter und Berufspendler, aber auch Hausfrauen und Hausmänner. Besonders besorgniserregend erscheint der Sachverhalt, dass neun von zehn Schülern (90\%) über Stress klagen und jeder dritte Schüler - analog zu den Ergebnissen bei den Erwachsenen - berichtet, permanent unter Leistungs- und Prüfungsdruck zu stehen. $\mathrm{Zu}$ ähnlich alarmierenden Befunden kommt die in den Jahren 2006-2010 von der Deutschen Sporthochschule Köln durchgeführten „Fit-Fürs-Leben“-Studie, in der mehr als 12.50o Personen im Alter von 6-25 Jahren hinsichtlich Bewegungs-, Ernährungs- und Lebensstilgewohnheiten sowie Gesundheit und körperliche Leistungsfähigkeit untersucht wurden (Leyk et al. 2012). Es zeigte sich, dass 52\% der Männer und 26\% der Frauen übergewichtig sind und 50\% der 25-jährigen Frauen und 59\% der Männer in der gleichen Altersgruppe rauchen. Zudem betreiben 37\% der Frauen und $27 \%$ der Männer nie oder nur selten Sport. Der alterspezifische Leistungsvergleich ergab außerdem, dass sich die 25-jährigen Personen auf dem körperlichen Leistungsniveau der 15-Jährigen befinden.

Angesichts der vorgestellten Befunde zu Stresserleben und mangelndem Gesundheitsverhalten ist es naheliegend, dass die Schulung von Introspektionsfähigkeiten ein wichtiger Ansatzpunkt ist, um sowohl Stresserleben als auch Gesundheitsverhalten langfristig zu modifizieren. 


\section{Achtsamkeitsbasierte Interventionen im Kontext von Medizin und Gesundheitsförderung}

Obwohl sich Bezüge zu Bewusstseinszuständen, die denen der Achtsamkeit nicht unähnlich sind, ohne Zweifel sowohl in psychologischen, psychiatrischen als auch psychotherapeutischen Systemen und Lehren - beispielhaft sei hier auf die Bedeutung der gleichschwebenden Aufmerksamkeit in Kontext der Psychoanalyse verwiesen - auffinden lassen (Ellenberger 1973; Kohls 2004), kann man die explizite Geburtstunde der Achtsamkeit als klinische Intervention in den späten 197oer-Jahren verorten. Zu dieser Zeit wurde von dem US-amerikanischen Molekularbiologen Jon KabatZin ein achtsamkeitsbasiertes Programm zur Verbesserung des Umgangs mit Belastung entwickelt, das er „Mindfulness-Based Stress Reduction Program“ (MBSR) nannte. MBSR ist ein standardisiertes, auf Selbsterfahrung aufbauendes psychoedukatives Achtwochenprogramm, durch das ein verbesserter Umgang mit Stress erlernt werden soll. Es beruht auf unterschiedlichen introspektiven Techniken wie BodyScan, formelle Meditation im Sitzen oder Gehen sowie spezifische Yoga-Übungen und kann ohne größeren Aufwand in unterschiedlichen Kontexten sowohl im Einzel- wie auch Gruppensettings implementiert werden kann (Kabat-Zinn 1996; 1998). Durch das MBSR-Programm soll eine spezifische Verbesserung der emotionalen Selbstregulationsfähigkeit erreicht werden, da durch das gezielte Einüben von Achtsamkeit nicht nur gelernt werden kann, den gegenwärtigen Moment „genauer“ wahrzunehmen, sondern auch die Fähigkeit entwickelt werden kann, Körperempfindungen, Gedanken, Gefühle zu betrachten und diese zu akzeptieren, ohne diese sofort kognitiv oder emotional bewerten zu müssen. Dies ist die Voraussetzung dafür, die Selbstregulationsfähigkeit zu verbessern. Das standardisierte MBSR-Basistraining besteht aus einem achtwöchigen Gruppenangebot von ca. 1,5 Stunden Dauer pro Woche, sowie zusätzlich einen ganzen Tag der Achtsamkeit. Zusätzlich wird von den Teilnehmern verlangt, dass sie täglich 30-45min eigenständig Achtsamkeitsübungen praktizieren. Die Achtsamkeitsmeditationsübungen zielen dabei darauf ab, einen inneren Ort der Aufmerksamkeit zu aktivieren, welcher es erlaubt, die Empfindungen/Gedanken des Moments wahrzunehmen, ohne diese sofort zu bewerten. Da die zugrundeliegenden Meditations- bzw. Introspektionstechniken überwiegend aus der Achtsamkeitstradition des Buddhismus - Vipassana - stammen, sind sie völlig von ihrem buddhistischen Kontext gelöst und folglich auch für Personen geeignet, die keine Affinität zu spirituell-religiösen Dimensionen oder andere religiöse Orientierungen haben. Mittlerweile wird das MSBR-Training in den USA an über 250 Kliniken und Gesundheitszentren zur Behandlung unterschiedlicher Krankheiten und Symptome erfolgreich eingesetzt. Das breite Anwendungsspektrum von MBSR wird auch durch mehrere Metaanalysen belegt, in der neben Studien mit geringerer methodischen Qualität auch qualitativ hochwertige empirische Studien analysiert und die Auswirkungen von MBSR auf unterschiedliche Gesundheitsparameter belegt wurden (Grossman et al. 2004; Eberth u. Sedlmeier 2012). Dabei zeigte sich über alle Studien hinweg eine durchschnittliche Wirksamkeit, der MBSR-Intervention, die durchaus mit psychotherapeutischen und pharmakologischen Therapien vergleichbar, wenn nicht sogar überlegen ist.

Durch das Eintrainieren einer wohlwollend-distanzierten Beobachterperspektive heben sich die Achtsamkeitsverfahren von anderen Introspektionstechniken ab, die auf einer Fokussierung oder willentlichen Lenkung des Bewusstseins beruhen(Sauer 
et al. 2011). Gelingen kann dies am Anfang vor allem durch eine Fokussierung auf den Atem, der Individuen helfen kann, ihr psychologisches Erleben in der Gegenwart zu verankern. Achtsamkeit kann und sollte jedoch nicht nur in den zurückgezogen Stunden der Kontemplation oder Meditation geübt werden, sondern auch innerhalb des Alltags. Dadurch wird Achtsamkeit nicht nur eine Praxis, sondern auch eine Lebenshaltung, und letztlich auch eine Art zu Sein. Weitere achtsamkeitsbasierte Verfahren stellen beispielweise der von dem Oxford-Psychologen Marc Williams und seinen Kollegen John Teasdale und Zindel Segal speziell zur Depressionsrückfallprophylaxe entwickelte Ansatz der „Mindfulness-Based Cognitive Therapy“ (MBCT) dar; ein weiteres frühes Beispiel ist die von Marsha Linehan zunächst als ambulantes Behandlungskonzept für Patientinnen mit Borderline-Störung und Suizidalitätsneigungen entwickelte „Dialektisch-Behaviorale Therapie“ dar. In Deutschland stoßen achtsamkeitsbasierte Interventionen im Kontext der Gesundheitsförderung zunehmend auf Interesse.

\section{Achtsamkeitsbasierte Interventionsprogrammen außerhalb von Medizin und Gesundheitsförderung}

Achtsamkeitsbasierte Interventionen stoßen zunehmend auf großes Interesse, so beispielsweise im Bereich der Pädagogik (Kaltwasser 2008; 2010), im Strafregelvollzug (Samuelson et al. 2007) oder auch im Kontext der Führungsforschung (Sauer et al. 2011; Sauer u. Kohls 2011). Bei diesen Ansätzen wird zumindest implizit davon ausgegangen, dass das Einüben von Achtsamkeit nicht nur gesundheitsbezogenen Nutzen haben kann, sondern sich auch positiv auf die Potenzialentfaltung in Bezug auf Dimensionen wie Lernen, Kreativität oder soziale und ethisch-moralische Kompetenz auswirken kann. Hierbei ist jedoch anzumerken, dass die wissenschaftliche Datenlage weit weniger umfassend ist, als im Fall der gesundheitswissenschaftlichen Auswirkungen.

Von unserer Arbeitsgruppe wurde beispielsweise kürzlich ein niederschwelliges achtsamkeitsbasiertes Programm, das speziell für Schüler einer fünften Klasse im Alter von 10-12 Jahren entwickelt wurde, im Rahmen einer Pilotstudie evaluiert (Kaltwasser et al. im Druck). Die Ergebnisse legen nahe, dass sich bereits durch ein dreimal die Woche durchgeführtes 15-minütiges Training die Konzentrationsleistung, das Stressempfinden und der soziale Interaktionsstil von Schülern positiv beeinflussen lassen.

In einer anderen Studie untersuchen wir gerade zusammen mit einem Kooperationspartner die Wirksamkeit von Achtsamkeit in der Arbeitswelt. Erste Ergebnisse sind vielversprechend und deuten darauf hin, dass sich nicht nur die Wahrnehmung der Belastung am Arbeitsplatz, Informationsverarbeitung sowie Entscheidungsfindungsprozesse verbessern lassen, sondern darüber hinaus auch der Umgang mit Kollegen und Kooperationspartnern verbessern kann, wenn Achtsamkeit in den Organisationen authentisch praktiziert wird.

Ein letzter Punkt berührt den in den letzten Jahren vielthematisierte Aspekt der unternehmerischen Sozialverantwortung und die Ausrichtung an ethischen und moralischen Handlungsprinzipien. In einer von der EU geförderten Studie wurde Wirksamkeit von Maßnahmen untersucht, mit denen Führungskräfte zu einer langfristigen und systematischen Umsetzung von unternehmerischer Sozialverantwortung ge- 
bracht werden sollen (Zollo et al. 2007). Es zeigte sich, dass die konventionellen Maßnahmen zur Umsetzung von unternehmerischer Sozialverantwortung, wie beispielsweise geleitete Gruppendiskussionen oder Einzelfallanalysen, nur wenig wirksam waren. Interessanterweise stellte sich jedoch heraus, dass Coachingprogramme mit introspektiven Komponenten sich als die wirksamsten Interventionen zur Ausbildung von ethischen und moralischen Verhaltensweisen erwiesen haben.

\section{Fazit: Funktionalisierung von Spiritualität oder Achtsamkeit als Self-Empowerment}

Achtsamkeit erfreut sich in vielen Bereichen zunehmender Popularität. Bei genauerer Betrachtung wendet sich das Konzept der Achtsamkeit gegen die in der weit verbreiteten Annahme, dass Bewusstsein lediglich ein Epiphänomen von neuronalen Hirnprozessen ist. Denn die wissenschaftlichen Erkenntnisse der letzten Jahrzehnte legen nahe, dass die Fähigkeit, das (Selbst-)Bewusstsein - im Sinne einer neuronalen Top-Down-Modellierung - als unabhängigen Prozess zu erleben, wirken zu lassen und - zumindest innerhalb gewisser Grenzen - zu modulieren, durch introspektive Techniken, wie beispielsweise der Achtsamkeitsmeditation, systematisch geschult werden kann.

Fasst man Achtsamkeitsschulung funktional als eine Form von "Self-Empowerment" auf, so stellt sich die Frage, ob es einen Unterschied macht, ob derartige Techniken als spirituelle Übungen verstanden werden, oder ob sie als Bewusstseintechniken in einem säkularisierten Kontext eingeübt werden. Die Frage im Zusammenhang mit der Funktionalisierung von Achtsamkeit haben auf dem Hintergrund der zunehmenden Popularität auch kritische Stimmen auf den Plan gerufen. So ist in einem kürzlich erschienen Blog der bekannten US-amerikanische Onlinezeitung Huffington Post mit dem scharfzüngigen Titel „Beyond McMindfulness" die Frage aufgeworfen worden, ob der ursprünglich in spirituellen Weltanschauungen ausgedrückte, spezifische Geist von Achtsamkeit durch die mit der in häufig in säkularisierter Form einhergehenden Popularisierung, Funktionalisierung und Ökonomisierung von achtsamkeitsbasierten Maßnahmen überhaupt in seiner Essenz aufrecht erhalten werden kann (Purser u. Loy 2013). Obwohl dies natürlich ein gewichtiges Argument ist, kann man meiner Ansicht nach im Sinne einer pragmatischen Sichtweise davon ausgehen, dass das Trainieren von Achtsamkeit letztlich dazu beiträgt, dass Menschen zu sich selbst finden können und ihre Gedanken, Einstellungen, Motivationen und Handlungen besser wahrnehmen können. Insofern bietet Achtsamkeit in meinen Augen eine große Möglichkeit zur Entwicklung, sowohl für den einzelnen Menschen als auch auf gesellschaftlicher Ebene.

\section{Literatur}

Arch II und Craske MG (2006) Mechanisms of mindfulness: Emotion regulation following a focused breathing induction. Behaviour Research and Therapy 44: 1849-1858

Bishop SR, Lau M, Shapiro S, Carlson L, Anderson ND, Carmody I, Segal ZV, Abbey S, Speca M, Velting D, Devins G (2004) Mindfulness: A proposed operational definition. Clinical Psychology: Science and Practice 11: 230-241

Cahn BR und Polich I (2006) Meditation States and Traits: EEG, ERP, and Neuroimaging Studies. Psychol Bull 132(2): 180-211

Davidson RJ, Kabat-Zinn I, Schumacher I, Rosenkranz M, Muller D, Santorelli SF, Urbanowski F, Harrington A, Bonus K, Sheridan JF (2003) Alterations in brain and immune function produced by mindfulness meditation. Psychosomatic Medicine 65: 564-570

Eberth ) und P Sedlmeier P (2012) The Effects of Mindfulness Meditation: A Meta-Analysis. Mindfulness 3(3): 174-189

Ellenberger HF (1973) Die Entdeckung des Unbewussten. Geschichte und Entwicklung der dynamischen Psychiatrie fon den Anfängen bis zu Janet, Freud, Adler und Jung. Bern, Huber 
Farb NA, Segal ZV, Mayberg H, Bean I, McKeon D, Fatima Z, Anderson AK (2007) Attending to the present: mindfulness meditation reveals distinct neural modes of self-reference. Social Cognitive and Affective Neuroscience 2(4): 313-322

Grossman P et al. (2004) Mindfulness based stress reduction and health: A meta-analysis. Journal of Psychosomatic Research 37: 35-43

Hölzel BK, Ott U, Gard T, Hempel H, Weygandt M, Morgen K, Vaitl D (2008) Investigation of mindfulness meditation practitioners with voxel-based morphometry. Social Cognitive and Affective Neuroscience Advance Access 3(1): 55-61

Kabat-Zinn I (1996) Gesund durch Meditation. Das grosse Buch der Selbstheilung. München, Barth.

Kabat-Zinn I. (1998) Im Alltag Ruhe finden: Das umfassende praktische Meditationsprogramm für alle Lebenslagen. Freiburg, Herder

Kaltwasser V (2008) Achtsamkeit in der Schule: Stille-Inseln im Unterricht: Entspannung und Konzentration. Weinheim, Beltz

Kaltwasser V (2010) Persönlichkeit und Präsenz. Achtsamkeit im Lehrerberuf. Weinheim, Beltz

Kaltwasser, V., Sauer, S., Kohls, N. (im Druck) Mindfulness in German Schools [MISCHO] - A specifically Tailored Training Program: Concept - Implementation and Empirical Results. Neuroscience, Consciousness and Spirituality. H. Walach, S. Schmidt and W. Jonas. Heidelberg, Springer. 2

Kohls N (2004) Außergewöhnliche Erfahrungen - Blinder Fleck der Psychologie? Eine Auseinandersetzung mit aussergewöhnlichen Erfahrungen und ihrem Zusammenhang mit geistiger Gesundheit. Münster, Lit-Verlag

Kohls $\mathrm{N}$ et al. (2009) Facets of Mindfulness - Results of an online study investigating the Freiburg mindfulness inventory. Personality and Individual Differences 46 46(2): 224-230

Lazar SW, Kerr CE, Wasserman RH, Gray JR, Greve DN, Treadway MT, McGarvey M, Quinn BT, Dusek JA, Benson H, Rauch SL, Moore Cl, Fischl B (2005) Meditation experience is associated with increased cortical thickness. Neuroreport 16(17): 1893

Leyk D, Rüther T, Witzki A, Sievert A, Moedl A, Blettner M, Hackfort D, Löllgen H (2012) Körperliche Leistung, Gewichtsstatus, Raucherquote und Sporthäufigkeit von jungen Erwachsenen. Dtsch Arztebl International 109(44): 737-745

Lutz A et al. (2008) Attention regulation and monitoring in meditation. Trends in Cognitive Sciences 12(4): 163-169

Purser R and Loy R (2013) Beyond McMindfulness The Blog. Huffington Post Religion, Huffington Post

Samuelson M et al. (2007) Mindfulness-Based Stress Reduction in Massachusetts Correctional Facilities. The Prison Journal $87(2): 254-268$

Sauer S et al. (2011) Mindful Leadership: Sind achtsame Führungskräfte leistungsfähigere Führungskräfte? Gruppendynamik und Organisationsberatung 42(4): 339-349

Sauer S and Kohls N (2011) Mindfulness in Leadership: Does Being Mindful Enhance Leaders' Business Success? Culture and Neural Frames of Cognition and Communication. S. Han and E. Pöppel. Heidelberg, Springer. 3: 287-308

Sauer S et al. (2011) Dialectics of Mindfulness: Implications for Western Medicine. Philosophy, Ethics, and Humanities in Medicine 6(1): 10

Sauer S et al. (2013) Assessment of Mindfulness: Review on State of the Art. Mindfulness 4(1): 3-17

Shapiro SL et al. (2006) Mechanisms of Mindfulness. Journal of Clinical Psychology 62(3): 373-386

Vaitl D (2005) Psychobiology of altered states of consciousness. Psychological Bulletin 131(1): p98-12711

Walach H et al. (2012) Mind-Body-Practices in Integrative Medicine. Religions 3: 50-81

Wittmann M (2009) Die Neuropsychologie der Zeit - Kognitive und emotionale Modulatoren der zeitlichen Erfahrung. Zeitschrift für Medizinische Psychologie 18(1): 28-39

Wittmann M (2011) Moments in time. Frontiers in integrative neuroscience 5(66)

Wittmann, M. (2013) The inner sense of time: how the brain creates a representation of duration. Nature Reviews Neuroscience 14(3): 217-223

Zollo M et al. (2007) Understanding and responding to societal demands on corporate responsibility (RESPONSE): final report, Island Press 


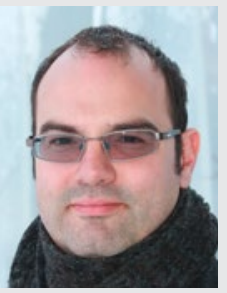

Prof. Dr. phil. Dr. habil. med. Niko Kohls

beschäftigt sich seit vielen Jahren mit dem Thema Achtsamkeit, Spiritualität und ihrem Zusammenhang mit Gesundheit und Lebensqualität. Er studierte in Jena und Freiburg Psychologie und wurde 2008 zum Scholar des US-amerikanischen Samueli Institute ernannt. An der Universität München hat er sich 2012 für Medizinische Psychologie habilitiert. Seit 2013 ist er an der Hochschule Coburg als Professor für Gesundheitswissenschaften tätig. 